\title{
Non-Neoplastic Genitourinary System Disorder
}

National Cancer Institute

\section{Source}

National Cancer Institute. Non-Neoplastic Genitourinary System Disorder. NCI

Thesaurus. Code C156664.

A non-neoplastic disorder that affects the genitourinary system. 